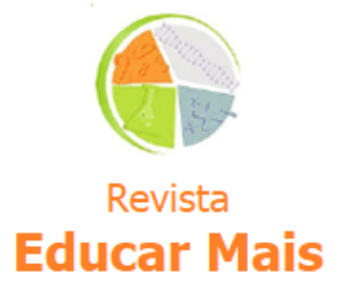

\section{A Utilização de Exercícios Intervalados de Alta Intensidade (HIIT) em Aulas de Ballet Fitness}

\section{The Use of High Intensity Interval Training (HIIT) in Ballet Fitness Classes}

Andréia Ambrósio-Accordi ${ }^{1}$; Iury de Almeida Accordi ${ }^{1}$

\title{
RESUMO
}

A inexistência de trabalhos acadêmicos sobre a aplicação de exercícios intervalados de alta intensidade (HIIT) no ballet fitness foi a principal motivação que justificou a elaboração desse trabalho. $O$ objetivo geral é descrever de que forma o HIIT é utilizado em aulas de Ballet Fitness aplicadas na Fundação de Cultura da Prefeitura Municipal de Caçador (Santa Catarina, Brasil). Optou-se por uma pesquisa qualitativa descritiva, enquadrada como um estudo de caso. Acompanhou-se a rotina das aulas de ballet fitness através de observação sistemática. Identificou-se a utilização de algum protocolo preestabelecido de HIIT através de observações sistemáticas. Os protocolos de HIIT utilizados foram analisados, através de entrevista estruturada, de modo a verificar se foram aplicados de forma planejada, de acordo com protocolos preexistentes, ou de forma intuitiva. Descreveu-se como é realizado o processo de planejamento das aulas. O HIIT aplicado nas aulas de ballet fitness observadas seguiu um planejamento intencional e não intuitivo, tratando-se de uma adaptação metodológica de protocolos preestabelecidos. Sugere-se algumas ações no intuito de minorar a evasão em geral e de aumentar o rendimento em particular.

Palavras-chave: Exercício; Técnicas de Exercício e de Movimento; Exercício Intermitente de Alta Intensidade.

\begin{abstract}
The lack of academic works on the application of high intensity interval training (HIIT) in ballet fitness was the main motivation that justifies the elaboration of this paper. The general aim is to describe how HIIT is used in ballet fitness classes offered at the Caçador City Hall Cultural Foundation (Santa Catarina, Brazil). We chose a qualitative descriptive research, classified as a case study. Ballet fitness class routines were followed through systematic observation. The use of a pre-established HIIT protocol was identified through systematic observations; through a structured interview, it was established whether the HIIT protocols used are applied in a planned way, according to pre-existing protocols, or in an intuitive way, in order to learn how the class planning process is carried out. The HIIT applied in the ballet fitness classes observed followed an intentional, non-intuitive planning, applying a methodological adaptation of pre-established protocols. Some actions are suggested in order to generally reduce absences and specifically increase performance.
\end{abstract}

Keywords: Exercise; Exercise Movement Techniques; High-Intensity Interval Training.

\footnotetext{
${ }^{1}$ IFSC - Instituto Federal de Educação, Ciência e Tecnologia de Santa Catarina, Joinville/SC - Brasil.
} 


\section{INTRODUÇÃO}

Desde os primórdios da civilização procura-se as melhores técnicas para maximizar as potencialidades do corpo humano, sejam elas físicas ou mentais. O poeta Romano Juvenal, no final do século I da era cristã imortalizou esse desejo no famoso adágio mens sana in corpore sano (mente sã num corpo são) (BRAUND, 2004, Sátira 10, p. 365, tradução nossa).

Já na Grécia antiga, havia uma preocupação com a manutenção de um corpo bem delineado e bem preparado fisicamente que ia, além das preocupações bélicas, ao encontro de ideais estéticos de beleza e de saúde (BARBOSA et al., 2011).

Ao longo da história, a prática dos exercícios físicos foi evoluindo, desde sua origem na Pré-História, afirmação na Antiguidade, dormência na Idade Média, fundamentação na Idade Moderna, sistematização no início da Idade Contemporânea até chegar aos dias de hoje: "mais desportiva, universalizada e plena de ecletismo" (RAMOS, 1983, p. 15).

Tamanho é o ecletismo, que Platonov e Bulatova (2003) dividem os exercícios físicos em cinco grupos, conforme a finalidade predominante da ação no desenvolvimento da motricidade no todo ou em alguns de seus aspectos: (1) exercícios de velocidade; (2) exercícios de força; (3) exercícios de coordenação; (4) exercícios para desenvolver a flexibilidade, e (5) exercícios para desenvolver a resistência.

Atualmente uma das modalidades mais procuradas é o treinamento resistido, por aumentar o potencial do aluno, dependendo dos métodos utilizados, para a prática de esportes e de atividades que exijam mais força, potência, controle e possivelmente resistência física (AABERG, 2002, p. xi). No entanto, muitas pessoas não se sentem atraídas a realizar esse tipo de treino, seja por causa da repetitividade demasiada dos exercícios, pela monotonia de suas práticas ou mesmo por desinteresse (PINHEIRO, 2010; LIZ; ANDRADE, 2016). Uma prática alternativa ao treinamento resistido para o público feminino é o ballet fitness.

O ballet fitness, modalidade criada no Brasil pela bailarina e professora de Educação Física Betina Dantas, conforme Ferreira e Santos (2016), mescla passos técnicos do ballet clássico na barra, com exercícios musculares no solo, objetivando condicionamento físico, alta queima calórica, melhora da postura e ainda tornar o corpo mais longilíneo e definido. Dessa forma, o ballet fitness utiliza cargas e repetições para trabalhar o corpo, com objetivos similares ao dos treinos de resistência (REBELO et al., 2017), envolvendo exercícios de força e de alta intensidade, os quais são também utilizados nos chamados treinos intervalados de alta intensidade.

O Treinamento intervalado de alta intensidade (HIIT, em inglês "high-intensity interval training") é composto por estímulos executados em alta, máxima ou supramáxima intensidade (GIBALA; MCGEE, 2008; BUCHHEIT; LAURSEN, 2013a).

Os estímulos executados em alta intensidade caracterizam-se pela orientação de esforços com níveis em torno de $90 \%$ do pico do consumo de oxigênio, podendo ser orientado por atividades executadas acima do segundo limiar ventilatório (GIBALA; MCGEE, 2008); já os estímulos executados em máxima ou supramáxima intensidade, também conhecido como atividades "all-out", caracterizam-se pela exigência do maior grau de intensidade que os participantes disponibilizam para a realização do exercício, ou então são protocolos desenvolvidos com o intuito de propor demandas de intensidades 
superiores ao máximo que os atletas desempenham em determinado parâmetro de avaliação (BUCHHEIT; LAURSEN, 2013a). Tais protocolos se mostraram capazes de taxar os sistemas de liberação de energia tanto anaeróbia quanto aeróbia quase que no máximo (TABATA et al., 1997).

O HIIT vem sendo utilizado atualmente seguindo diversos protocolos, que levam em conta a duração do ciclo do treino, composto pela duração do estímulo e tempo de recuperação, bem como os números de ciclos no decorrer da sessão (MACHADO et al., 2017). Estes autores citam, por exemplo, que protocolos de HIIT podem abarcar sessões que duram entre 4 e 45 minutos, com duração do estímulo entre 20 segundos e 4 minutos e com um tempo de recuperação entre 10 segundos e 4 minutos.

Sabe-se que o HIIT é uma estratégia eficiente em termos de tempo para induzir numerosas adaptações metabólicas geralmente associadas com o treinamento de resistência tradicional (GIBALA; MCGEE, 2008) e, na ausência de equipamento de ginástica, pode ser bem adequado para a manutenção da aptidão em pessoal moderadamente treinado (GIST et al., 2015).

Notou-se que em vários momentos das aulas de Ballet Fitness aplicadas na Fundação de Cultura da Prefeitura Municipal de Caçador (Santa Catarina, Brasil), utilizavam-se sequências de HIIT. A partir dessa observação surgiu uma indagação que norteará o trabalho: essas sequências de HIIT seguem um protocolo previamente planejado ou são estabelecidas de forma intuitiva pela professora?

Dessa forma, o objetivo geral desse trabalho é relatar de que forma o HIIT é utilizado nas aulas de Ballet Fitness aplicadas na Fundação de Cultura da Prefeitura Municipal de Caçador (Santa Catarina, Brasil). Tem-se como objetivos específicos: realizar uma revisão bibliográfica a respeito do uso de HIIT em aulas de Ballet Fitness, acompanhar a rotina dessas aulas; identificar quais são os protocolos de HIIT utilizados; verificar de que forma esses protocolos são aplicados, e conhecer como é realizado o processo de planejamento das aulas.

\section{PROCEDIMENTOS METODOLÓGICOS}

Optou-se por uma pesquisa qualitativa descritiva que, conforme Gil $(2002$, p. 42) tem como objetivo primordial a "descrição das características de determinada população ou fenômeno", no presente caso, a descrição das formas como o HIIT é aplicado nas aulas de Ballet Fitness da Fundação de Cultura da Prefeitura de Caçador.

Com base nos procedimentos técnicos utilizados, a presente pesquisa se enquadra como um estudo de caso, que, conforme Yin (2001, p. 31) trata-se de uma "investigação empírica que investiga um fenômeno contemporâneo dentro de seu contexto da vida real, especialmente quando os limites entre o fenômeno e o contexto não estão claramente definidos". No caso do presente estudo, não há um limite claro entre onde as técnicas do Ballet Fitness dão lugar à utilização de protocolos de HIIT e nem se esses protocolos são aplicados de forma planejada ou intuitiva. É justamente a esse tipo de situação que Gil (2002, p. 54) se refere ao citar a utilização do estudo de caso com o propósito de "descrever a situação do contexto em que está sendo feita determinada investigação".

Realizou-se uma extensa revisão bibliográfica através do portal de periódicos CAPES, Google Acadêmico, PubMed, SPORTDiscus e referências cruzadas de outros artigos. Utilizou-se as palavraschave "HIIT", "treinamento intervalado", "interval training", "balé", Ballet", Ballet fitness". 
A rotina das aulas de ballet fitness foi acompanhada através de observação sistemática que, conforme Marconi e Lakatos (2007, p. 90) é aquela que é realizada sob condições controladas e objetiva responder a propósitos preexistentes. De modo a reduzir a tendenciosidade e falta de representatividade da amostra, as observações foram realizadas em múltiplas ocasiões (YIN, 2016, p. 167): nas duas turmas existentes e em todos os horários de aula, que eram segundas e quartas das $18 \mathrm{~h} 30$ min às $19 \mathrm{~h} 30$ min e terças e quintas das 7 h 30 min às 8 h 30 min, entre 20 de março e 9 de maio de 2017.

O acompanhamento das aulas de ballet fitness foi relatado através de uma descrição que detalhou características das turmas, como tamanho da turma, quantidade de alunas por barra e duração dos diversos momentos das aulas. $\mathrm{O}$ relato da rotina das aulas foi complementado por fotografias $\mathrm{e}$ filmagens que serviram como fontes de dados para análise.

Para identificar se algum protocolo preestabelecido de HIIT foi seguido, realizaram-se observações sistemáticas ao longo das aulas onde foram anotadas em uma planilha as variáveis manipuláveis do HIIT (conforme Del Vecchio et al., 2014b): duração do estímulo, intervalo de recuperação e número de séries. Por fim, verificou-se, com base nos dados compilados, se as aulas de ballet fitness seguiram a um ou a vários protocolos de HIIT e se esses protocolos se encaixam dentro de algum protocolo já descrito na bibliografia existente.

De modo a verificar se os protocolos de HIIT utilizados nas aulas de ballet fitness são aplicados de forma planejada, de acordo com protocolos preexistentes, ou de forma intuitiva, realizou-se uma entrevista estruturada seguindo um roteiro previamente estabelecido (MARCONI; LAKATOS, 2007, p. 93-94), com as seguintes perguntas direcionadas à professora de ballet fitness: (1) dos blocos de exercícios preparados para a aula, quantos são destinados ao HIIT?; (2) em que momentos da aula esses blocos de HIIT são utilizados?; (3) qual o tempo de duração das sessões (blocos de exercícios) somente de HIIT?; (4) qual a duração de cada exercício de HIIT?; (5) qual o tempo de recuperação entre os exercícios de HIIT?; (6) qual a base teórica utilizada para a aplicação do HIIT nas aulas?; (7) existe um protocolo de HIIT seguido para as aulas?; (8) se sim, houve alguma modificação nesse protocolo para a aplicação nas aulas e em qual das variáveis manipuláveis?

Para conhecer como é realizado o processo de planejamento das aulas, também foi realizada uma entrevista estruturada seguindo um roteiro previamente estabelecido com as seguintes perguntas direcionadas à professora de ballet fitness. (1) você segue o estilo de aula de Betina Dantas ou criou o seu próprio estilo?; (2) quantas horas diárias você ocupa para a preparação das aulas?; (3) qual a inspiração para a preparação das aulas? (4) o planejamento das aulas para todas as turmas é o mesmo?; (5) se não é o mesmo, qual o diferencial?; (6) em média, quantos blocos de exercícios (sessões) são preparados por aula?

A entrevistada assinou Termo de Consentimento Livre e Esclarecido, concedendo sua anuência, de acordo com a resolução 466 de 2012 do Conselho Nacional de Saúde do Ministério homônimo, sobre diretrizes e normas regulamentadoras de pesquisa envolvendo seres humanos (BRASIL, 2012). A pesquisa foi analisada pelo Comitê de Ética em Pesquisa do Instituto Superior e Centro Educacional Luterano Bom Jesus/IELUSC e, de acordo com o parecer 2.908.757 foi considerada adequada para aplicação de acordo com os critérios éticos. 
As observações e entrevistas realizadas durante o trabalho de campo foram compiladas e classificadas de modo a formar uma base de dados que seguiu uma ordem conforme os objetivos específicos que foram determinados para o presente projeto.

\section{RESULTADOS E DISCUSSÃO}

\subsection{O uso do HIIT em aulas de Ballet Fitness}

Na busca de artigos que associassem o uso de HIIT em aulas de Ballet ou Ballet Fitness, encontrouse um grande número de artigos publicados a respeito do estudo do HIIT nas mais variados grupos de pessoas e modalidades esportivas, como obesos e/ou portadores de síndrome metabólica (IRVING et al., 2008; ARAUJO et al.; 2012; HEYDARI et al., 2012; ALKAHTANI et al., 2013; DEL VECCHIO et al., 2014a; KEATING et al., 2014); pessoas com sobrepeso (MARRA et al., 2003; 2005); pessoas em grupo de risco para doenças cardiometabólicas (GIBALA et al., 2012); pessoas treinadas ou atletas de elite (alto desempenho) (GIBALA et al., 2012; BUCHHEIT; LAURSEN, 2013a, b; ALMEIDA; TOLEDO, 2014; KILEN et al., 2014); pessoas idosas (BILLAT, 2001); crianças e adolescentes (BILLAT, 2001); corredores (BILLAT, 2001; BUCHHEIT; LAURSEN, 2013a, b; HOTTENROTT et al., 2012); ciclistas (LAURSEN; JENKINS, 2001); e praticantes de esportes coletivos (IAIA et al., 2009; BUCHHEIT; LAURSEN, 2013a, b). No entanto, apenas um artigo foi encontrado sobre as aplicações do HIIT para o treinamento de fitness em geral (SCHOENFELD; DAWES, 2009) e nada referente à aplicação do HIIT ao ballet fitness.

\subsection{Rotina das aulas de Ballet Fitness}

As turmas de Ballet Fitness eram compostas por um público exclusivamente feminino com idades entre 21 e 49 anos (média de 33,62, +/- 8,31 d.p.). A turma da manhã era composta por 30 alunas e a noturna por 35 alunas.

As aulas de Ballet Fitness não utilizam caneleiras e nem haltere. A proposta é usar exatamente o peso do corpo para melhorar a força, a flexibilidade e o equilíbrio. Também não há uma vestimenta específica para a prática do Ballet Fitness, podendo-se utilizar collants, shorts, ou outras roupas confortáveis. No entanto, indica-se o uso de uma sapatilha que pode ser de pano ou que imite uma meia.

A sala era composta por três barras grandes de $4 \mathrm{~m}$ e uma barra menor de $3 \mathrm{~m}$ onde eram realizados a maioria dos exercícios. Cada barra de $4 \mathrm{~m}$ pode comportar até cinco alunas e a de $3 \mathrm{~m}$ tem uma lotação de até três alunas. Na maior parte das aulas as barras ficavam com sua lotação completa.

A sala onde eram realizadas as aulas de Ballet Fitness era grande e bem arejada, com muitas janelas e ventiladores em todas as paredes. Havia também um espelho na parte frontal que ia de parede a parede.

Houve uma alta rotatividade quanto à composição das turmas, pois existia uma fila de espera e a aluna que faltasse duas aulas perdia a vaga e cedia seu lugar para a próxima da fila.

As aulas começavam em um ritmo mais lento para aquecimento e evoluíam para ritmos mais intensos que contavam com exercícios de resistência ou HIIT e terminavam com exercícios de flexibilidade e alongamento. 
Foram registrados dois formatos de aula, em circuito e em bloco. Quando a aula era em circuito, formavam-se quatro circuitos e dentro desses, dois exercícios eram destinados ao HIIT. Nas aulas executadas em bloco, realizava-se uma sequência de ballet clássico, exercícios de barra, exercícios de centro e de fitness. Os exercícios de fitness eram compostos por dez blocos, sendo que, em média, cinco desses eram destinados ao HIIT. A rotina observada em aula conferiu com a que foi relatada pela professora na entrevista.

\subsection{Protocolos de HIIT utilizados nas aulas de Ballet Fitness}

Em seu relato, a professora afirmou que começou utilizando o protocolo Tabata em suas aulas, mas atualmente acha difícil seguir um protocolo em particular devido à dificuldade de trabalhar com grandes turmas onde há uma alta desproporcionalidade entre o preparo das alunas, que vão desde fisicamente inativas até altamente preparadas. "É uma dificuldade seguir um protocolo ideal que se encaixe na realidade de todas as pessoas", afirmou. Portanto, o protocolo, pelas razões expostas acima foi modificado na duração das sessões, no tempo de recuperação e no tempo de estímulo.

De acordo com o que foi observado e com o relato coletado, dentro da duração da aula de ballet fitness as séries de HIIT eram aplicadas em vários momentos intercalados. As séries de HIIT seguiram o seguinte padrão: duração do estímulo entre 20 e 32 s; intervalo de recuperação entre 4 e 10 s; número de séries de 3 a 4, o que coincidiu com o relatado. Comparou-se o padrão detectado com os três principais protocolos de HIIT utilizados em aulas de fitness: Tabata 1 e 2 (TABATA et al., 1997) e Gibala (GIBALA e MCGEE, 2008). Como pode ser observado na Tabela 1, o padrão utilizado para as séries de HIIT durante as aulas de ballet fitness pode ser considerado uma mistura dos protocolos Tabata 1 e 2, onde observa-se parâmetros de duração do estímulo e intervalo próximos do Tabata 1 e parâmetros de duração do estímulo e número de séries próximo do Tabata 2.

Tabela 1 - Principais protocolos de HIIT utilizados em aulas de fitness comparados com o padrão de HIIT aplicado nas aulas de ballet fitness da Fundação Municipal de Cultura de Caçador, observado entre março e maio de 2017.

\begin{tabular}{ccccc}
\hline Variável & \multicolumn{3}{c}{ Protocolo } & Padrão das aulas de \\
\cline { 2 - 4 } Ballet Fitness
\end{tabular}

Fontes: Tabata et al. (1997); Gibala; McGee (2008), e os autores deste trabalho (2017).

De acordo com a entrevista realizada, o referencial teórico utilizado pela professora para a aplicação de HIIT em suas aulas de ballet fitness é baseado no referencial teórico das linhas de pesquisa do Professor Sérgio Gregório da Silva do Departamento de Educação Física da Universidade Federal do Paraná (ver, por exemplo, os trabalhos de FERREIRA, 2017; FOLLADOR et al., 2018).

Ainda conforme a entrevista, os blocos de HIIT são utilizados intercalados dentro das aulas. A aula inicia com um aquecimento, onde não há HIIT; no segundo exercício, dependendo da temperatura, 
já se começa com um pequeno trabalho de HIIT (o que para algumas alunas já é um grande esforço por estarem iniciando e com pouco preparo e para outras trata-se de um exercício mais leve); a partir do terceiro exercício tenta-se trazer um HIIT mais forte, que continua no quarto exercício; no quinto exercício procura-se diminuir a frequência cardíaca com um HIIT mais leve, finalizando com um bloco sem HIIT . Observou-se que as aulas começam em um ritmo mais lento para aquecimento e evoluem para ritmos mais intensos que podem ser com exercícios de resistência ou HIIT e termina com exercícios de flexibilidade e alongamento.

De acordo com a entrevista e o que foi observado, existem dois formatos de aula: em circuito e em bloco. Quando era aplicado o formato de aula em circuito, formavam-se quatro circuitos e dentro desses, dois exercícios eram destinados ao HIIT. Quando as aulas eram executadas em bloco, executava-se uma sequência de ballet clássico, exercícios de barra, exercícios de centro e de fitness.

Os exercícios de fitness eram compostos por dez blocos, sendo que, em média, cinco desses destinados ao HIIT.

Conforme relatado, o planejamento das aulas para as duas turmas é o mesmo, e segue o método Betina Dantas. A base para a preparação das aulas é buscada através de vários recursos, como aulas na internet, cursos pagos e livros. A professora não informou, no entanto, quais as suas fontes bibliográficas ou de internet.

Não foi observada nenhuma mudança no padrão relatado de aula quanto à frequência e intensidade do HIIT. Por sua vez, vários estudos apontam a necessidade de uma troca de protocolos ao longo do treinamento, diminuindo o tempo de recuperação para aumentar o VO2max e a capacidade anaeróbica (BILLAT, 2001; DEL VECCHIO et al., 2014a), ou mesmo a manipulação de variáveis de determinado protocolo para adaptá-lo a uma modalidade esportiva em específico (IAIA et al., 2009; BUCHHEIT, 2010; BUCHHEIT; LAURSEN, 2013a; HERODEK et al., 2014).

Também pode ser desejável o uso de uma mistura de treinos com intensidade supramáxima e outros com intervalos mais prolongados de modo a reduzir a monotonia de uma sessão de treinamento (ESFARJANI; LAURSEN, 2007).

Ao longo do período de observação, pode-se acompanhar todo o processo de aplicação das aulas de Ballet Fitness e como o HIIT é incorporado a essas. Foi possível distinguir em quais momentos eram aplicados o HIIT ou exercícios sem ele e foi possível apurar a veracidade do depoimento da professora em relação ao planejamento de suas aulas, que refletiram fidedignamente o que fora aplicado na prática.

Além disso, constatou-se a dificuldade relatada pela professora de usar um protocolo em resposta ao questionamento em função do desnivelamento da turma, que era composta por alunas que iam desde um nível inicial de treinamento até outras com um nível mais avançado, que inclusive praticavam outras atividades físicas.

Outro fato observado foi a alta rotatividade, que se deu principalmente entre as alunas mais novas, aparentemente as menos preparadas, que desistiam entre a segunda e terceira semanas de aula. Havia um núcleo central de alunas mais antigas, que deveriam ser, de acordo com uma observação inicial, as mais preparadas e as mais assíduas. 
Essa heterogeneidade relacionada à experiência e ao maior tempo de treino pode ser considerada o principal fator responsável pela desistência de alunas novatas, que não conseguem seguir o ritmo das mais antigas. Del Vecchio et al. (2014a) frisaram que diferentes protocolos de HIIT visando a um aumento do potencial motivacional e da eficiência temporal podem aumentar a adesão aos programas de exercícios. Tais protocolos, porém, devem ser progressivos, começando com uma intensidade submáxima até chegarem em uma intensidade supramáxima conforme o progresso individual (DEL VECCHIO et al., 2014a). Para tanto, seria necessário que as turmas de ballet fitness fossem divididas em, pelo menos, duas categorias, iniciantes e veteranas.

\section{CONSIDERAÇÕES FINAIS}

O HIIT foi aplicado em vários momentos durante as aulas de ballet fitness das turmas da Fundação Municipal de Cultura de Caçador, SC, seguindo um planejamento intencional e não intuitivo, tratandose de uma mistura de protocolos preestabelecidos.

Sugere-se, no entanto, a divisão das turmas em iniciantes e veteranas, para que possa haver uma efetiva progressão no rendimento das alunas e uma alternância de protocolos de HIIT ao longo das aulas. Ambas as ações objetivando uma menor evasão em geral e um maior rendimento em particular.

Além disso, o presente relato se reveste de uma importância acadêmica adicional, pois trata-se, de acordo com a revisão bibliográfica extensiva realizada, não só da primeira contribuição acadêmica sobre a utilização do HIIT em aulas de ballet fitness, mas também o primeiro trabalho relatando a prática do ballet fitness em si.

Constatou-se a necessidade, em virtude da absoluta falta de estudos acadêmicos a respeito da prática do ballet fitness, de estudos adicionais que envolvam aspectos fisiológicos do rendimento de praticantes dessa modalidade, bem como traçar o perfil socioeconômico e motivacional das praticantes, de modo a identificar as causas que as levaram à escolha da modalidade.

\section{REFERÊNCIAS}

AABERG, Everett. Conceitos e técnicas para o treinamento resistido. Barueri: Manole, 2002. $223 \mathrm{p}$.

ALKAHTANI, Shaea. A.; KING, Neil A.; HILLS, Andrew P.; BYRNE, Nuala M.. Effect of interval training intensity on fat oxidation, blood lactate and the rate of perceived exertion in obese men. SpringerPlus, v. 2, n. 532, 2013. Disponível em: http://www.springerplus.com/content/2/1/532. Acesso em: 19 mai. 2019.

ALMEIDA, Fábio Eduardo; TOLEDO, Thiago Fernando Contijo de. Efeitos do treinamento aeróbio de moderada intensidade e intervalado de alta intensidade (HIIT) sobre a composição corporal de homens treinados. Muzambinho:IF Sul de Minas, 2014. Trabalho de Conclusão de Curso (Bacharelado) - Faculdade de Educação Física, Instituto Federal de Educação, Ciência e Tecnologia do Sul de Minas Gerais 2014.

ARAUJO, Ana Carolina Corte de; ROSCHEL, Hamilton; PICANÇO, Andreia Rossi; PRADO, Danilo Marcelo Leite do; VILLARES, Sandra Mara Ferreira; PINTO, Ana Lúcia de Sá; GUALANO, Bruno. Similar Health Benefits of Endurance and High-Intensity Interval Training in Obese Children. PLoS ONE, v. 
7, n. 8, n. $\quad$ e42747, 2012. Disponível em: http://journals.plos.org/plosone/article?id=10.1371/journal. pone.0042747. Acesso em 19 mai. 2019.

BARBOSA, Maria Raquel; MATOS, Paula Mena; COSTA, Maria Emília. Um olhar sobre o corpo: o corpo ontem e hoje. Psicologia e Sociedade, v. 23, n. 1, p. 24-34, 2011. Disponível em: http://www.scielo.br/pdf/psoc/v23n1/a04v23n1.pdf. Acesso em: 19 mai. 2019.

BILLAT, L. Veronique. Interval Training for Performance: a Scientific and Empirical Practice. Special Recommendations for Middle- and Long-Distance Running. Part II: Anaerobic Interval Training. Sports Medicine, v. 31, n. 2, p. 75-90, 2001.

BRAUND, Susanna. H. (ed.; tradutora). Juvenal and Persius. Cambridge, London: Harvard University Press, 2004. 536 p. (Loeb Classical Library).

BRASIL. Resolução No 466, do CNS/MS sobre Diretrizes e Normas Regulamentadoras de Pesquisa envolvendo seres humanos, de 12 de dezembro de 2012. Disponível em: http://conselho.saude.gov.br/resolucoes/2012/Res0466.pdf. Acesso em: 19 mai. 2019.

BUCHHEIT, Martin. The 30-15 Intermittent Fitness Test: 10 years review. Myorobie Journal, V. 1, 2010. Disponível em: http://www.cardioc.eu/wp-content/uploads/2016/12/buchheit-30-15ift-10-yrsreview-2000-2010.pdf. Acesso em: 19 mai. 2019.

BUCHHEIT, Martin.; LAURSEN, Paul B. High-Intensity Interval Training, Solutions to the Programming Puzzle. Part II: Anaerobic Energy. Neuromuscular Load and Practical Applications. Sports Medicine, v. 43, n. 10 , p. $927-954,2013 a$.

BUCHHEIT, Martin.; LAURSEN, Paul B. High-Intensity Interval Training, Solutions to the Programming Puzzle. Part I: Cardiopulmonary Emphasis. Sports Medicine, v. 43, n. 5, p. 313-338, 2013b.

DEL VECCHIO, Fabrício Boscolo; GALLIANO, Leony Morgana; COSWIG, Victor Silveira. Aplicações do exercício intermitente de alta intensidade na síndrome metabólica. Revista Brasileira de Atividade Fisica e Saúde, v. 18, n. 6, p. 669-687.

DEL VECCHIO, Fabrício Boscolo; GALLIANO, Leony Morgana; PICANÇO, Luan Merseburger; RIBEIRO, Yuri Salenave. Treinamento intervalado de alta intensidade: definição de termos e variáveis manipuláveis. In: DEL VECCHIO, Fabrício Boscolo; RIBEIRO, Yuri Salenave; PICANÇO, Luan Merseburger; GALIANO, Leony Morgana (Eds.) Exercício intermitente: estado da arte e aplicações práticas. Pelotas: OMP Editora, 2014b. p 11-16.

ESFARJANI, Fahimeh; LAURSEN, Paul. B. Manipulating high-intensity interval training: effects on VO2max, the lactate threshold and $3000 \mathrm{~m}$ running performance in moderately trained males. Journal of Science and Medicine in Sport, v. 10, p. 27-35, 2007.

FERREIRA, Sandro dos Santos. Efeito do exercício autosselecionado e intervalado de alta intensidade nas respostas fisiológicas, perceptuais e afetivas nas mulheres com excesso de gordura corporal. Tese (Doutorado em Educação Física) - Curitiba: UFPR, 2017. Disponível em: https://acervodigital.ufpr.br/handle/1884/50308. Acesso em: 19 mai. 2019.

FOLLADOR, Lucio; ALVES, Ragami C.; FERREIRA, Sandro dos S.; BUZZACHERA, Cosme F.; ANDRADE, Vinícius F. dos S.; GARCIA, Erick D. S. de A.; OSIECK, Raul; BARBOSA, Sara C.; OLIVEIRA, Letícia M. de; SILVA, Sérgio G. da. Physiological, perceptual, and affective responses to six high-intensity interval training protocols. Perceptual and Motor Skills, v. 125, n. 2, p. 329-350, 2018. 
GIBALA, Martin J.; MCGEE, Sean L. Metabolic Adaptations to Short-term High-Intensity Interval Training: A Little Pain for a Lot of Gain? Exercise and Sport Sciences Reviews, v. 36, n. 2, p. 5863, 2008.

GIBALA, Martin J.; LITTLE, JonathaP.; MACDONALD, Maurren; HAWLEY, John A. Physiological adaptations to low-volume, high-intensity interval training in health and disease. Journal of Physiology, v. 590, n. 5, p. 1077-1084, 2012.

GIL, Antônio Carlos. Como elaborar projetos de pesquisa. 4. ed. São Paulo: Atlas, 2002. 176 p.

GIST, N. H.; FREESE, E. C.; CURETON, K. J. Effects of Low-Volume, High-Intensity Whole-Body Calisthenicson Army ROTC Cadets. Military Medicine, v. 180, n. 5; p. 492-498, 2015. 